\title{
Application Article \\ Phase Pattern Calibration for Interferometric Applications in Spaceborne SAR Systems
}

\author{
Markus Bachmann, Marco Schwerdt, Gabriel Castellanos Alfonzo, and Dirk Schrank \\ German Aerospace Center (DLR), Oberpfaffenhofen, 82234 Weßling, Germany \\ Correspondence should be addressed to Markus Bachmann; markus.bachmann@dlr.de
}

Received 5 April 2013; Accepted 30 September 2013

Academic Editor: Ulrich Nickel

Copyright ( 2013 Markus Bachmann et al. This is an open access article distributed under the Creative Commons Attribution License, which permits unrestricted use, distribution, and reproduction in any medium, provided the original work is properly cited.

\begin{abstract}
SAR is a widely used technique to acquire images for geoscience and earth observation applications. Active phased array antennas are commonly used in spaceborne SAR systems. For certain modes and applications, it is necessary to know the phase behavior of these phased array antennas. For applications utilizing the different polarization channels for interferometry, the phase difference between the polarizations needs to be calibrated very accurately as it is the main evaluation parameter. Also for single-pass interferometric missions, the difference between the two antennas in terms of phase gradients is of major importance. This paper demonstrates for the first time the usage of phase patterns in an operational interferometric SAR mission. It describes why these phase patterns are required and how they are used to fulfill the different goals of the missions. Then, the mathematical model to derive the phase of the antenna patterns is shown. Finally, the paper explains how the antenna patterns are calibrated in order to minimize their residual errors and describes in detail the measurements performed for this calibration and verification.
\end{abstract}

\section{Introduction}

Spaceborne Synthetic Aperture Radar (SAR) missions essentially contribute to geoscience for a better understanding of our global environment. With its all day, all night, and all weather imaging capabilities on the one hand and a high resolution on the other, SAR is an ideal tool to monitor these processes affecting our environment regularly and to help understanding the effects behind. In addition, SAR interferometry enables the generation of high resolution digital elevation models (DEMs) adding to the information content provided by nominal SAR images.

As SAR is an active system, it requires a transmit antenna to send radar pulses. The echoes are received with the same or a different antenna, stored and processed into SAR images. The antenna of such a system is commonly realized as an active phased array antenna. Like every antenna, it shows a certain radiation characteristic, the so-called antenna pattern, which is visible in the uncorrected SAR images. During processing, the influence of the antenna pattern is removed.

For nominal single polarized SAR images, only the influence of the amplitude of the pattern is relevant. This is described in detail in $[1,2]$. Since the SAR imaging principle relies on coherently integrating the echoes from multiple transmit pulses spread over time and space, precise phase alignment between and within pulses is a critical component of the system performance.

Interferometric imaging exploits the phase difference between two slightly offset, highly colinear SAR images to infer terrain height. INSAR applications require very precise joint phase characterization of the transmit and reveice antenna patterns as a function of look angle.

This paper is divided thematically as follows: Section 2 describes the applications utilizing the phase information. Section 3 gives an overview about the challenges of phase pattern determination and calibration and Section 4 derives the mathematical background of the phase patterns. Section 5 provides the calibration approach and the results for the different phase pattern calibration aspects.

\section{Phase Pattern Applications}

Two main applications utilize the phase information of the SAR phased array antenna. 
2.1. Polarimetric SAR Interferometry and the Dual Receive Antenna Mode. Polarimetric SAR Interferometry (PolInSAR) [3] is a widely used technique to determine soil and moisture of areas on the Earth's surface, tree heights, or vegetation growth. Multiple images acquired with two or even four polarizations during one acquisition are combined to extract the required information content. For PolInSAR the accurate and stable phase information between the polarization channels is essential since it must be corrected during processing to remove systematic perturbations that degrade system performance.

For PolInSAR, the accuracy of the phase knowledge needs to be better than $5 \mathrm{deg}$. This was the requirement for the TerraSAR-X mission, which performs SAR imaging for scientific and commercial customers and was launched in 2007. The same value was required for the L-band system ALOS/PALSAR [4]. The ALOS/PALSAR mission showed that it is possible to calibrate the phase balance down to an imbalance of $0.61 \mathrm{deg}$ with a standard deviation of $2.66 \mathrm{deg}$ [5].

One important parameter for PolInSAR is the crosscoupling between different polarizations. This isolation of the cross-talk for TerraSAR-X was already measured and is better than $34 \mathrm{~dB}[6,7]$.

A topic closely related to PolInSAR is the usage of the Dual-Receive Antenna (DRA) capabilities of TerraSAR-X. For the DRA mode, the receive antenna is split in two halves in azimuth direction. The signal from both parts are received by two separate receiver chians and stored separately. Hence, two simultaneously acquired images with a small along-track baseline in azimuth direction are obtained. These images can be evaluated for applications like Along Track Interferometry (ATI) for Ground Moving Target Indication (GMTI) [8]. In the same way as for PolInSAR, well calibrated phase patterns are also important for the phase compensation in DRA acquisitions [9].

2.2. Bistatic Imaging. Multisatellite bistatic formations are currently becoming more and more popular in spaceborne SAR imaging. TanDEM-X, the first bistatic SAR formation, has been in orbit since 2010. For bistatic systems it is quite important to know the phase gradient for each beam in transmit and receive direction and especially individually for both satellites. Therewith, the monostatic and bistatic phase patterns of the satellites are derived in order to coherently combine the images for interferometric applications [10]. For bistatic missions using interferometry to determine heights on the Earth, the influence of the phase from the two receiving systems needs to be known and compensated for.

The main goal of the TanDEM-X mission is the derivation of a global Digital Elevation Model (DEM) [11]. The accuracy requirement of this mission in terms of phase accuracy can be derived from the overall mission requirement for the relative height error. The $90 \%$ relative height error of the DEM in a $1^{\circ} \times 1^{\circ}$ cell shall not exceed $2 \mathrm{~m}$. The two satellites in space build a spaceborne interferometer with a height of ambiguity down to $30 \mathrm{~m}$, where the height of ambiguity corresponds to one phase cycle of 2 pi or $360^{\circ}$. Hence, to achieve a relative height error of $2 \mathrm{~m}$ [11], the phase error of the overall system may not exceed $12 \mathrm{deg}$. Since receiver noise over areas with low backscatter return eats up the greatest part of this error budget, errors associated with the antenna pattern should be lower than $3 \mathrm{deg}$.

\section{Challenges}

The usefulness of an antenna model for nominal SAR imaging is given in detail in [1]. Here, only the main considerations important to the antenna phase patterns are described.

3.1. Great Number of Beams. Actual SAR missions host very flexible instruments. With different modes, hundreds of antenna beams can be used to acquire images for dedicated applications and purposes. Any of these beams can be used for polarimetry and interferometry. New imaging modes and antenna beams can even be added after the launch. These new beams were not measured on ground prior to launch. An accurate antenna model is essential to mathematically determine the antenna gain and phase patterns for each beam and thereby ensure in situ system performance.

3.2. Accuracy Requirement. The accuracy requirements of actual missions and their tight schedules make the measurement of all antenna patterns in orbit infeasible. Hence, the antenna model for TerraSAR-X was validated by on-ground measurements leaving only a few beams to be measured on orbit. A great advantage of the antenna model is that it can be adjusted over mission life time to reflect in-situ performance. Establishing different correction factors during commissioning phase and operational phase enables the adjustment and extension of the model to further improve system performance.

3.3. Need for Antenna Phase Patterns. A common way to account for the phase behaviour of an antenna is the definition and determination of a "phase center," that is, a geometrical point from which the antenna pattern appears to emanate. However, this phase center is only easily determined for a horn antenna. For a phased array antenna, individual phase and amplitude taper values can be applied to each subelement. For an untapered beam, the resulting phase variation is still very smooth over the main lobe of a beam shown by the red graph in Figure 1. It could still be possible, with a certain inaccuracy, to describe the phase of the entire array with a single phase center.

In the Shuttle Radar Topography Mission (SRTM) mission, for example, only beams steered in phase but not tapered in amplitude were used to realize the ScanSAR acquisitions [12] in C-band and the single swath acquisitions in X-band. The influence of the phase was analysed [13]. It was found that constant phase offsets were sufficient to describe the phase behaviour and no range angle dependent pattern was applied.

The antenna beams of TerraSAR-X are optimized to provide SAR images with high radiometric accuracy. For this purpose, the antenna pattern is steered and formed using individual phase and amplitude tapering coefficient. Figure 2 shows these excitation coefficients of an exemplary Stripmap beam. In the transmit direction no amplitude taper 


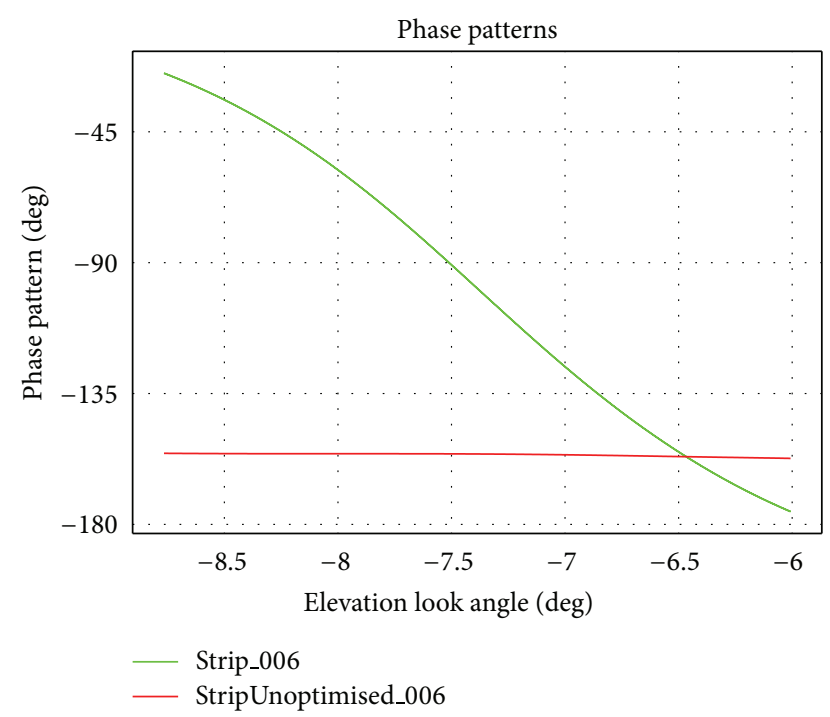

FIGURE 1: Phase patterns of a tapered (green) and an untapered (red) beam.

is applied due to performance considerations. In receive up to $-13 \mathrm{~dB}$ of the amplitude taper is used to attenuate the signal and thereby to optimize the performance; this is clearly visible in Figure 2. This amplitude taper for Stripmap beams results in a large phase variation over the beam width. This is again shown in Figure 1 where the phase gradient of the tapered beam is shown in green. Depending on the beam and the corresponding excitation coefficients, phase gradients of more than 180 deg can occur.

3.4. Relative and Absolute Phase Derivation. In practice, only a relative phase calibration $\Delta \varphi=\varphi_{2}-\varphi_{1}$ is possible. For an absolute measurement of the phase, the orbit would have to be known within a fraction of the wave length. For an X-band system with a wave length of $3.1 \mathrm{~cm}$, using GPS measurements, the orbital position of the satellite is determined on the order of 5 to $10 \mathrm{~cm}$, which is at least an order of magnitude too coarse. Additionally, the influence of the atmosphere makes it very difficult to determine absolute phase.

For PolInSAR, however, the images for each polarization are taken quasi at the same time. The polarization is switched from pulse to pulse and the orbit trajectory is very stable over the SAR integration time. Hence, the influence of the orbit on the phase difference is very nearly zero and can be safely ignored.

For interferometric imaging on the other hand, the absolute baseline between the two antenna centers is critically important. Because of the orbital stability, this relative vector can be determined on the order of $1 \mathrm{~mm}$ (1 sigma) with spacebased systems using double differential GPS measurements $[14,15]$. Therefore, it is accurate enough for absolute height determination, provided that at least one ground control point is available, that is, a pixel or region in the image, with a precise knowledge of its absolute height and geolocation is available to calibrate the absolute height.

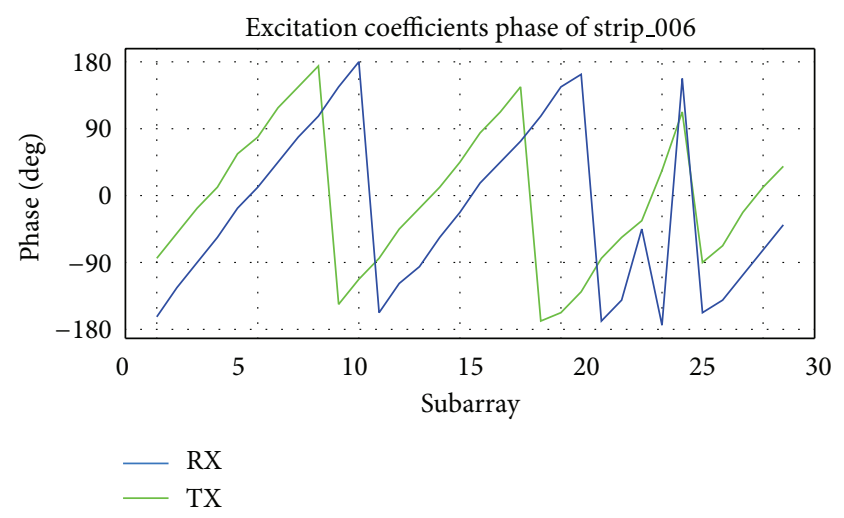

(a)

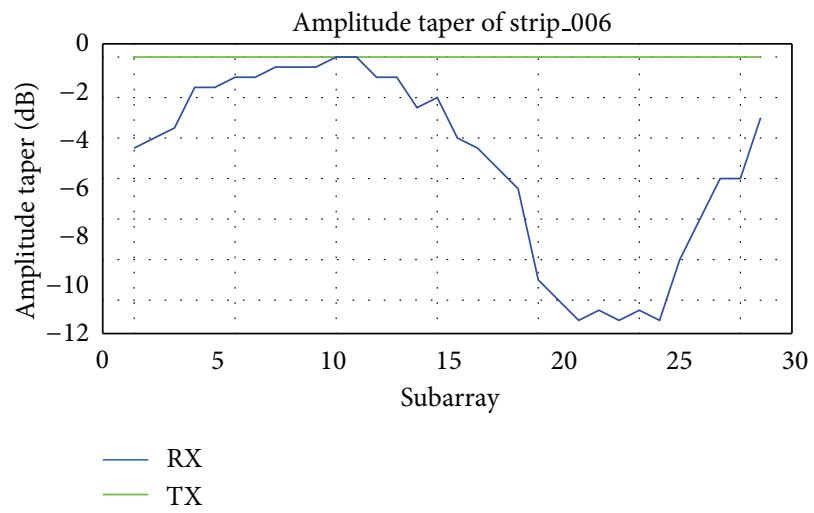

(b)

Figure 2: Excitation coefficients of a tapered stripMap-Beam.

\section{Antenna Pattern Modeling}

4.1. Antenna Model Equation. The mathematics behind the antenna model was developed in the context of the TerraSAR$\mathrm{X}$ mission in cooperation between EADS Astrium $\mathrm{GmbH}$, Friedrichshafen, and the DLR. It was extended by DLR to apply the phase pattern concept for the TanDEM-X mission.

The antenna model itself mathematically calculates radiation patterns by the superposition of four inputs:

(i) radiation patterns of the single-antenna elements mounted in the final array configuration (to cover mutual coupling effects) measured on ground (prelaunch), the so-called embedded subarray patterns,

(ii) beam excitation coefficients (amplitude and phase) of each individual transmit/receive module (TRM),

(iii) exact geometrical dimensions of the array antenna including the vertical distances between the subarrays,

(iv) the current state of the SAR instrument including drifting and/or failed TRMs. 
For active phased array antennas, the radiated pattern $F_{\text {beam }}$ is calculated by $[16,17]$

$$
\begin{array}{r}
\vec{F}_{\text {beam }}(\varepsilon, \alpha)=\sum_{m=0}^{M-1} \sum_{n=0}^{N-1}\left(\vec{C}_{\mathrm{SA}, m n}(\varepsilon, \alpha) \cdot a_{m n} \cdot E_{\mathrm{SA}, m n}\right. \\
\cdot e^{j k \sin \varepsilon \cos \alpha(-((N-1) / 2)+n) \Delta y} \\
\left.\cdot e^{j k \cos \varepsilon \sin \alpha(-((M-1) / 2)+m) \Delta x}\right),
\end{array}
$$

where $\varepsilon$ and $\alpha$ are the desired elevation and azimuth angle, $N$ and $M$ are the amount of rows and panels of the subarrays, and $\Delta x$ (panels) and $\Delta y$ (rows) are the intersubarray distances. The wave number $k$ includes the centre frequency $9.65 \mathrm{GHz}$ of the system by the relation of $k=2 \pi / \lambda$. The embedded subarray patterns $C_{\mathrm{SA}}$, the excitation coefficients $a_{m n}$, and the error matrix $E_{\mathrm{SA}}$ are described in the next section.

4.2. Antenna Model Inputs. The embedded subarray patterns $C_{\mathrm{SA}}$ comprise the physically measured complex radiation characteristics of the individual subarray elements. The embedded pattern of one subarray mounted within the array antenna describes the radiation characteristic of this subarray. In this way, mutual coupling effects and phase distortions are incorporated in the measurements. For the nominal acquisition, mismatch and gain are smooth over the bandwidth; therefore, only the embedded patterns at centre frequency need to be incorporated in the antenna model.

As input for the antenna model, all embedded subarrays of one panel, which is 32 subarrays in a column, were measured by Astrium. The patterns of the embedded patterns at different panels but situated at the same row are very similar to each other, independent from the position of the panel. The comparison of the measured patterns of whole panels showed that it is possible to use only the embedded patterns of one panel and substitute the others using their measured amplitude and phase offsets. These embedded patterns must be known with high accuracy, as they are one main input of the model. The measurements must be more accurate than the required setting accuracy of the TRMs, which is $0.5 \mathrm{~dB}$ in amplitude and $5 \mathrm{deg}$ of phase; this corresponds to about $0.4 \mathrm{~mm}$ of baseline separation.

The commanded complex excitation coefficients are given by $a_{m n}$. These coefficients are provided in terms of amplitude and phase values row- and column-wise. For each beam commanded, one set of values is put in a common table. This table is available on board of the satellite to be applied to form the beam for each acquisition. It can be updated if necessary, for example, in case of contingencies.

Finally, the error matrix $E_{\mathrm{SA}}$ describes drifted or failed antenna elements. These are determined via a TRM characterization method [18]. With the method, the actual state of the TRMs is determined evaluating dedicated orthogonal code sequences routed through the TRMs sequentially.

To obtain the complete two-way antenna patterns, the equation is evaluated for transmit and receive separately. Both, the excitation laws and the error matrix are different for transmit and receive. In case of the excitation coefficients, the discrimination between transmit and receive allows greater flexibility for beam steering, as from signal-to-noise ratio point of view, the use of an amplitude taper is only reasonable in the receive path. Since the radar signals travel through the TRMs on different paths, for transmit via the high power amplifier and in receive through the low noise amplifier, the error matrix coefficients for the two paths will be different.

The accuracy of the antenna model depends strongly on the measurement accuracy of the embedded subarray patterns and on the stability of the instrument.

4.3. Phase Pattern Derivation, Calibration, and Compensation. To derive and generate the phase pattern $\varphi_{\text {beam }}$ in elevation direction, (1) is evaluated:

$$
\varphi_{\text {beam }}(\varepsilon)=\arctan \left(\vec{F}_{\text {beam }}(\varepsilon)\right)
$$

For nominal acquisitions, the antenna is constantly steered to azimuth boresight. Hence, the phase variation in azimuth direction remains stationary. During phase calibration of the polarization, a slight polynomial dependency was recognized from the measurements. This dependency can be compensated by the relation

$$
\varphi_{\text {polcor }}(\epsilon)=\left(\varphi_{c 1}^{2} \varepsilon^{2}+\varphi_{c 3} \varepsilon+\varphi_{c 3}\right)+\varphi_{\text {beam }}(\varepsilon)
$$

with $\varphi_{c 1}, \varphi_{c 2}$, and $\varphi_{c 3}$ being determined by the polarimetric calibration described in Section 5.1.

Especially for bistatic acquisitions an adaption of the different phase origins of both satellites $\varphi_{\text {sat }}$ has to be established by a phase correction:

$$
\varphi_{\text {cor }}(\epsilon)=\varphi_{\text {sat }}+\varphi_{\text {polcor }}(\varepsilon)
$$

which is determined by the interferometric calibration described in Section 5.3.

The resulting phase pattern is now unwrapped at phase wraps in the pattern. These phase wraps occur at nulls in the amplitude pattern, for example, between the main lobe and the first side lobe, where the phase jumps by 180 deg:

$$
\varphi_{\text {unwrap }}=\operatorname{unwrap}\left(\varphi_{\text {cor }}\right) \text {. }
$$

The corrected phase pattern is then stored and provided to the SAR processor, where the phase dependency of the image is compensated $[19,20]$. The correction is applied on the single look slant range complex SAR (SSC) data. A reference DEM is used to determine the local incidence angles. These are then calculated back into antenna geometry, to correctly apply the phase pattern.

\section{Calibration and Verification Results}

\subsection{Polarization Dependency}

5.1.1. Purpose. For PolInSAR, the difference between the two polarizations is utilized. As the complete antenna pattern is formed by the superposition of embedded subarray patterns of the individual elements, these embedded patterns are the 


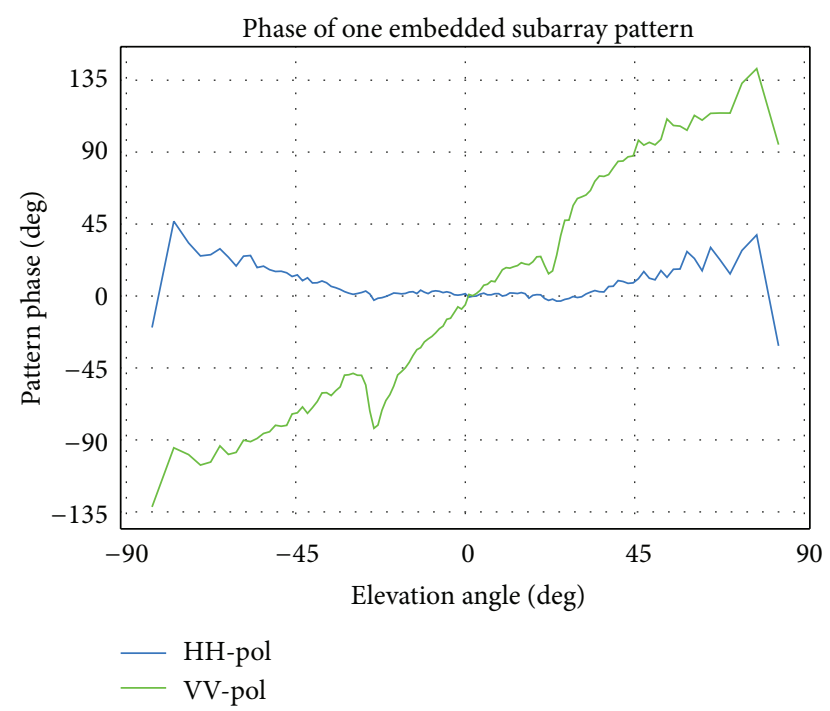

FIgure 3: Phase deviation between $\mathrm{HH}$ and VV polarization for one subarray.

main influence for the polarization dependency. As depicted in Figure 3 the patterns show an elevation angle dependent phase gradient, which differs between $\mathrm{HH}$ and VV. This dependency makes it necessary to not only correct one offset between the polarizations but also provide a correction pattern covering each whole swath.

5.1.2. Approach. The impact of the phase deviations on the whole antenna was measured by an in-flight measurement campaign. Corner reflectors deployed in a calibration field around Oberpfaffenhofen were used as targets for several dual-pol acquisitions with different incidence angles from near to far range. In dual-pol acquisitions the polarization is switched from pulse to pulse either in transmit or receive direction or a combination of both. Hence, they provide the advantage that both polarizations are acquired from almost the same time and from the same orbital position and penetrating the same atmosphere under the same weather conditions. By comparing both resulting images, the phase difference can be determined very accurately.

5.1.3. Results. Initially, a variation of about $10 \mathrm{deg}$ was observed over the elevation angle range. From these initial measurements, the polynomial correction described by (3) was derived. This correction is applied in the antenna model to adjust the embedded patterns.

The final results can be seen in Figure 4(a). The deviation between $\mathrm{HH}$ and VV polarization can be calculated by the antenna model very precisely. Figure 4(b) shows the deviation between $\mathrm{HH}$ and $\mathrm{VV}$ compared between the antenna model and the physical measurements. It reveals a standard deviation of only 1.69 deg (1 sigma).

By design, the polarization switch of the radar system is situated between the radiator and the TRM. Hence, all active components are the same for both polarizations. Only the passive radiators are different for both polarizations. However, they are recirocal. This reciprocity could be confirmed

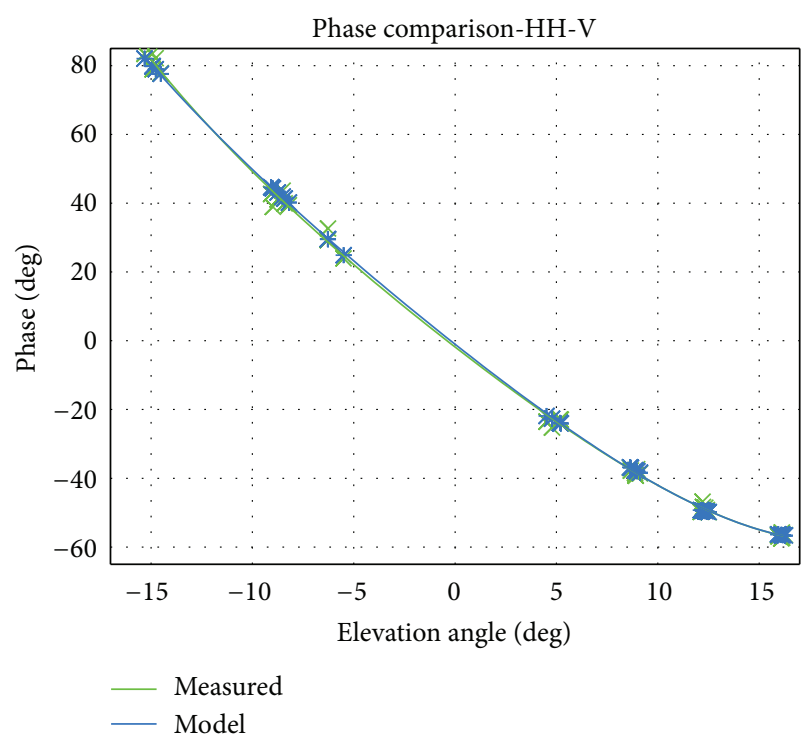

(a)

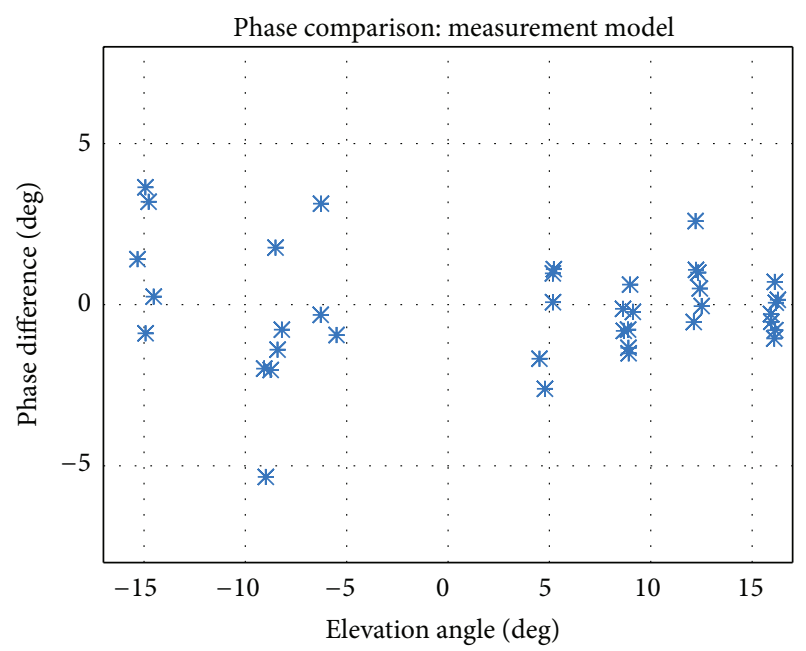

(b)

FIGURE 4: (a) Verification of the polarization dependent phase deviation: Measurements versus Antenna Model, (b) Deviation between HH-VV of the model and the measurements.

by evaluating dedicated Dual-Pol data takes with polarization combinations like $\mathrm{HH} / \mathrm{HV}$ and $\mathrm{VV} / \mathrm{HV}$. Hence, the variation of all combinations including $\mathrm{HV}$ or $\mathrm{VH}$ polarized phase is equal or lower than the one for $\mathrm{HH} / \mathrm{VV}$.

\subsection{Phase Pattern Shape}

5.2.1. Purpose. The calibration and verification of the phase pattern shape is required to ensure that differently excited beams do not introduce an additional error into the system.

In TanDEM-X acquisitions, beams with and without amplitude taper are mixed for the acquisition of the DEM. In TanDEM-X, the Earth will be covered twice. The beams used for the second acquisition are shifted by half the beam width compared to the first acquisition. By this, areas with lower 
antenna gain at the edges of the main lobe in one coverage are combined with a high antenna gain in the center of the main lobe from the other coverage. To keep the gain in the main lobe as high as possible, the beams are not amplitude tapered to flatten them. However, for difficult terrain like deserts, also optimized beams can be used to acquire additional data. These, in contrast, are the nominal Stripmap beams. Amplitude tapering is used to flatten and broaden the near beams to obtain sufficient performance over the whole swath. The beam to compare with is tapered in amplitude and shows a different taper in phase over the 32 subarrays in elevation on the TerraSAR-X satellite (Figure 1). Especially due to the taper in amplitude, the phase shows a steep gradient of about $50 \mathrm{deg}$ per degree look angle, while the untapered beam is almost flat (compare Figure 1). An error in the modeling of this pattern would lead to phase trends in the interferograms and the DEMs.

By design, the antennas of both satellite systems are very similar. Hence, the phase behaviour is also very similar. However, if several modules of one antenna fail or drift, the phase behaviour would change severely. For this purpose, the quantification of the joint phase patterns incorporating both satellites is critically important for the system to meet its mapping specifications.

5.2.2. Approach. To evaluate the accuracy of the phase pattern shape, two DEMs acquired with different beams formed with two difference excitation coefficient sets can be compared. For this purpose, a beam covering the same coverage area but with different excitation coefficients and with no amplitude taper was designed and uploaded to the instrument

Two acquisitions are taken from the same orbital location, imaging the same test area on Earth, but with a lag of one repeat cycle (11 days). To study the accuracy of the antenna model, two beams illuminating the same swath but with different excitation coefficients are used. The different excitation coefficients lead to completely different shapes of the antenna pattern. The first beam is only steered by phase coefficients which lead to an antenna pattern with typical shape. The second beam is optimized to form a very flat pattern by using both phase and amplitude taper. By the use of the amplitude taper, also the embedded patterns of different rows are weighted differently. Hence, the comparison would reveal a mistaken usage of these inputs for phase pattern generation. The generated DEMs (see Figure 5 for an example of the test site near Quandong Airport, Australia) are annotated in lat/lon coordinates, which makes it easy to build the difference image of them. The difference image can then be integrated in flight direction to generate a DEM difference graph which is shown in Figure 6.

5.2.3. Results. To derive the corresponding phase error associated with the DEM difference in phase units, the height of ambiguity of the two acquisitions must be taken into account. For the first acquisitions this was $h_{\mathrm{amb}}=128 \mathrm{~m}$ (untapered beam), and $h_{\mathrm{amb}}=131 \mathrm{~m}$ (tapered beam) for the second acquisition. With a mean $h_{\mathrm{amb}}$ of $129.5 \mathrm{~m}$ the peak-to-peak height error $\Delta h$ transforms by

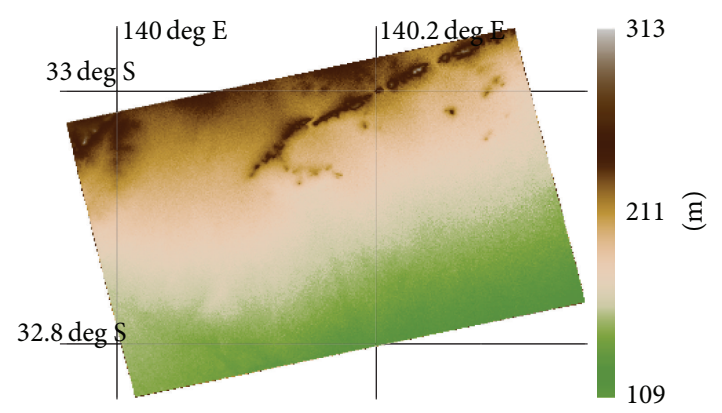

FIgURE 5: DEM of the Quandong test site.

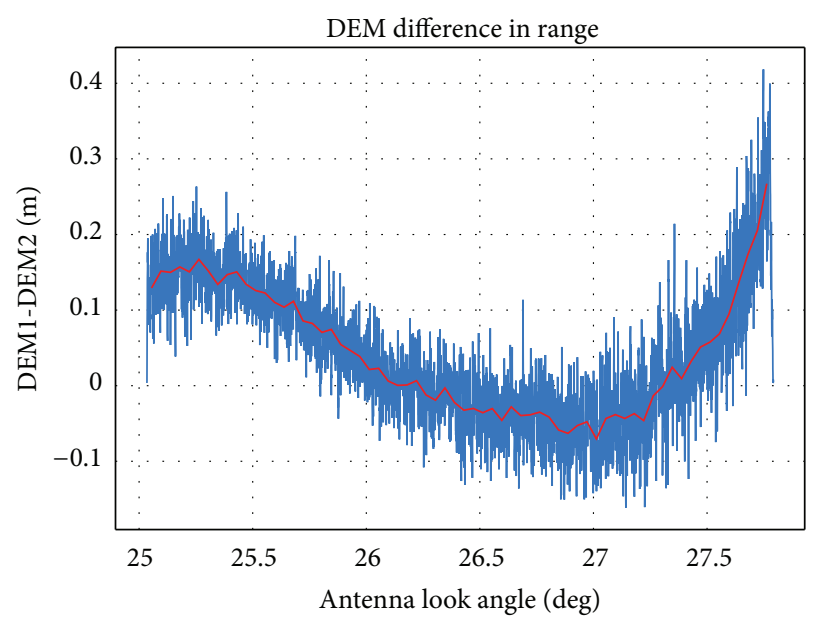

FIGURE 6: Resulting phase pattern deviation in flight direction of the difference between the two DEMs.

$$
\varphi_{\mathrm{err}}=\frac{\Delta h}{h_{\mathrm{amb}}} 2 \pi
$$

into a peak-to-peak phase error of $\varphi_{\text {err }}=0.85 \mathrm{deg}$ and a standard deviation of only $0.39 \mathrm{deg}$. Hence, the pattern shape has been accurately calculated by the antenna model.

\subsection{Beam to Beam Dependency}

5.3.1. Purpose. Proper phase compensation of the beam to beam dependency is important to remove systematic tilts in the DEM in range direction. As nine beams are required to cover the whole ground range of $240 \mathrm{~km}$ of one orbit at the equator, no shift between the beams or an overall trend should be present.

5.3.2. Approach. To verify the influence of the phase patterns from beam to beam, all individual DEMs acquired so far can be evaluated. For each DEM, the deviation from a reference DEM is calculated. In our case this reference DEM is an SRTM DEM globally improved using the height information of ICES at elevation datasets [21]. It is obvious that the accuracy of SRTM is lower than what would be expected for this verification. However, the SRTM swaths with $240 \mathrm{~km}$ ground range are much larger than TanDEM-X swaths with $30 \mathrm{~km}$. The SRTM DEM is combined from ascending and 
descending acquisitions. Due to the different inclinations of the Space Shuttle (SRTM) and TerraSAR-X/TanDEM$\mathrm{X}$, the swaths of both overlap randomly. In this way, a trend in the SRTM DEM is not systematically traced to the difference between SRTM and TanDEM-X average DEM heights. It has to be mentioned that the DEM scenes are not DEM-calibrated at this point, where DEM calibration means the compensation of range and azimuth trends for each individual scene separately with respect to the others.

5.3.3. Results. More than 50000 independently processed DEM scenes of the global DEM acquisition, each covering an area of $30 \mathrm{~km} \times 50 \mathrm{~km}$ and about 10 million pixel, were evaluated using their mean height difference to the improved SRTM. Only the nine nominal TanDEM-X beams were evaluated covering an incidence angle range from 30 deg to $48.5 \mathrm{deg}$, which is the nominal incidence angle range for TanDEM-X DEM acquisitions.

To relate both satellites against each other, an interferometric calibration [22] was performed before determining the phase offsets between both satellites to be considered in (5). Here, one global phase offset per satellite that is valid for all acquisitions was derived and applied to calibrate the system which is explained in [20]. The intension of the present paper for this topic was to ensure that there is no residual trend over the beams caused by the antenna model.

Figure 6 shows the mean heights of all scenes plotted over the nine beams. The mean height of ambiguity for the scenes is about $60 \mathrm{~m}$. With this, the phase accuracy calculated from the maximal deviation from Figure 7 of a height error of $\pm 0.24 \mathrm{~m}$ using (6) is $\pm 1.5 \mathrm{deg}$. No further systematic trend is evident in the residuals, and hence the interferometric calibration has reached its limits.

\section{Conclusion}

The knowledge of the phase gradient over the antenna look angle is of fundamental importance for certain applications in SAR image acquisition. For high performance SAR instruments with a multitude of beams and modes, modeling the antenna phase gradient offers significant performance advantages over purely instrumentally derived patterns.

The paper shows the generation and application of phase patterns for PolInSAR, for the Dual-Receive Antenna Mode and for a fully operational bistatic SAR system in space. The phase patterns are generated by an antenna model and calibrated in orbit with very high accuracy.

Considering the stringent requirement for phase pattern accuracy in high performance SAR systems, different applications have to be distinguished. Comparing the different polarizations over the whole look angle range, the phase difference between the phase patterns of each polarization channel is modeled with an accuracy better than 1.69 deg ( 1 sigma). An accuracy of 0.85 deg could be achieved comparing different amplitude taper and excitation coefficients of the antenna look angle used for the phase patterns. The average dependency from beam to beam is also better than $\pm 1.5 \mathrm{deg}$ comparing the nominal nine TanDEM-X beams.

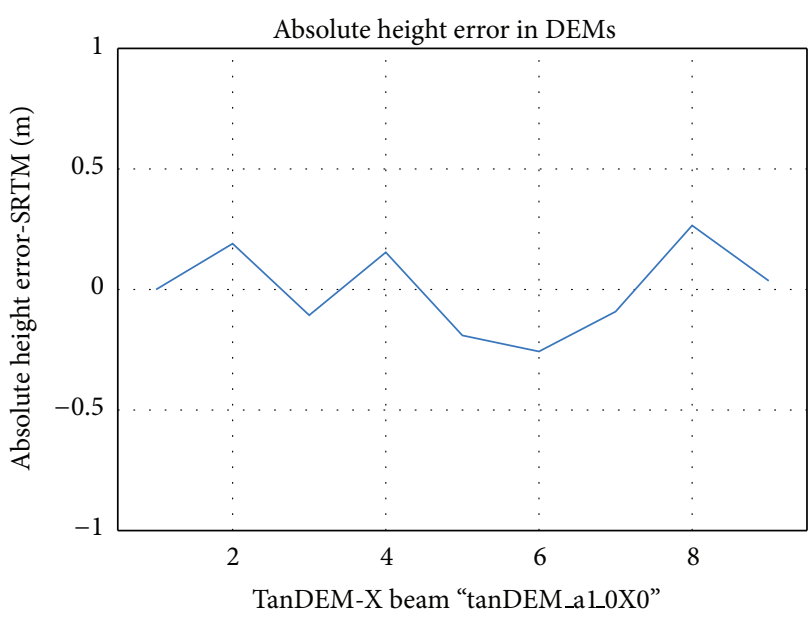

FIGURE 7: Beam to beam dependency of the phase pattern.

\section{Acknowledgments}

The work related to the TanDEM-X project mentioned in this paper is partly funded by the German Federal Ministry for Economics and Technology (Förderkennzeichen $50 \mathrm{EE}$ 1035). The authors would like to thank the Calibration Team for deploying Corner Reflectors for the analysis and the SAR Processor/Interferometric Processor Team for processing the acquisitions and DEMs used for the analyses. Special thanks go also to the HR team for providing the infrastructure for the analyses. We also would like to thank the Astrium $\mathrm{GmbH}$ that built these very stable and reliable satellites.

\section{References}

[1] M. Bachmann, M. Schwerdt, and B. Bräutigam, “TerraSAR-X antenna calibration and monitoring based on a precise antenna model," IEEE Transactions on Geoscience and Remote Sensing, vol. 48, no. 2, pp. 690-701, 2010.

[2] M. Schwerdt, B. Bräutigam, M. Bachmann, B. Döring, D. Schrank, and J. Hueso Gonzalez, "Final TerraSAR-X calibration results based on novel efficient methods," IEEE Transactions on Geoscience and Remote Sensing, vol. 48, no. 2, pp. 677-689, 2010.

[3] K. P. Papathanassiou and S. R. Cloude, "Single-baseline polarimetric SAR interferometry," IEEE Transactions on Geoscience and Remote Sensing, vol. 39, no. 11, pp. 2352-2363, 2001.

[4] A. Rosenqvist, M. Shimada, N. Ito, and M. Watanabe, "ALOS PALSAR: a pathfinder mission for global-scale monitoring of the environment," IEEE Transactions on Geoscience and Remote Sensing, vol. 45, no. 11, pp. 3307-3316, 2007.

[5] M. Shimada, O. Isoguchi, T. Tadono, and K. Isono, "PALSAR radiometric and geometric calibration," IEEE Transactions on Geoscience and Remote Sensing, vol. 47, no. 12, pp. 3915-3932, 2009.

[6] I. Hajnsek, K. Papathanassiou, T. Busche et al., "Data quality and scientific analysis of fully polarimetric TerraSAR-X data," in Proceedings of the POLInSAR, 2011.

[7] M. Schwerdt, J. Hueso Gonzalez, M. Bachmann, D. Schrank, C. Schulz, and B. Döring, "Monostatic calibration of both 
TanDEM-x satellites," in Proceedings of the 30th IEEE International Geoscience and Remote Sensing Symposium (IGARSS '10), pp. 2636-2639, July 2010.

[8] M. Gabele, B. Brautigam, D. Schulze, U. Steinbrecher, N. TousRamon, and M. Younis, "Fore and aft channel reconstruction in the TerraSAR-X dual receive antenna mode," IEEE Transactions on Geoscience and Remote Sensing, vol. 48, no. 2, pp. 795-806, 2010.

[9] M. Schwerdt, D. Schrank, M. Bachmann, C. Schulz, B. Döring, and J. Hueso Gonzalez, "TerraSAR-X Re-calibration and dual receive antenna campaigns performed in 2009," Proceedings of the 8th European Synthetic Aperture Radar Conference (EUSAR '10), 2010.

[10] P. A. Rosen, S. Hensley, R. Ian Joughin et al., "Synthetic aperture radar interferometry," in Proceedings of the Geoscience and Remote Sensing Symposium (IGARSS '02), 2000.

[11] G. Krieger, A. Moreira, H. Fiedler et al., "TanDEM-X: a satellite formation for high-resolution SAR interferometry," IEEE Transactions on Geoscience and Remote Sensing, vol. 45, no. 11, pp. 3317-3340, 2007.

[12] S. Hensley, P. Rosen, and E. Gurrola, “The SRTM topographic mapping processor," in Proceedings of the Intenational Geoscience and Remote Sensing Symposium (IGARSS '00), pp. 11681170, July 2000.

[13] M. Zink and D. Geudtner, "Calibration of the interferometric XSAR system on SRTM," in Proceedings of the IEEE International Geoscience and Remote Sensing Symposium (IGARSS '99), pp. 227-229, July 1999.

[14] J. Hueso Gonzalez, J. Walter Antony, M. Bachmann, G. Krieger, M. Schwerdt, and M. Zink, "Tests of the TanDEM-X DEM calibration performance," in Proceedings of the International Geoscience and Remote Sensing Symposium (IGARSS '12), 2012.

[15] O. Montenbruck, M. Wermuth, and R. Kahle, "GPS based relative navigation for the TanDEM-X mission-first flight results," in Proceedings of the 23rd International Technical Meeting of the Satellite Division of the Institute of Navigation, pp. 2797-2807, September 2010.

[16] M. Bachmann, M. Schwerdt, B. Bräutigam, B. Grafmüller, A. Herschlein, and J. L. Álvarez-Pérez, "The TerraSAR-X antenna model approach," in Proceedings of the 2nd International ITG Conference on Antennas (INICA '07), Munich, Germany, March 2007.

[17] C. A. Balanis, Antenna Theory: Analysis and Design, John Wiley \& Sons, 2nd edition, 1997.

[18] B. Brautigam, M. Schwerdt, and M. Bachmann, "An efficient method for performance monitoring of active phased array antennas," IEEE Transactions on Geoscience and Remote Sensing, vol. 47, no. 4, pp. 1236-1243, 2009.

[19] T. Fritz and M. Bachmann, "L1b product phase pattern correction,” Tech. Rep. TX-GS-TN-3031, TerraSAR-X Ground Segment, 2010.

[20] T. Fritz, C. Rossi, N. Yague-Martinez, F. Rodriguez-Gonzalez, M. Lachaise, and H. Breit, "Interferometric processing of TanDEM-X data," in Proceedings of the IEEE International Geoscience and Remote Sensing Symposium (IGARSS '11), pp. 2428-2431, Vancouver, Canada, July 2011.

[21] M. Huber, B. Wessel, D. Kosmann et al., "Ensuring globally the TanDEM-X height accuracy: analysis of the reference data sets ICESat, SRTM and KGPS-tracks," in Proceedings of the IEEE International Geoscience and Remote Sensing Symposium (IGARSS '09), pp. II769-II772, July 2009.
[22] M. Bachmann, J. Hueso Gonzalez, G. Krieger, M. Schwerdt, J. Walter Antony, and F. De Zan, "Calibration of the bistatic TanDEM-X interferometer," in Proceedings of the 9th European Synthetic Aperture Radar Conference (EUSAR '12), 2012. 

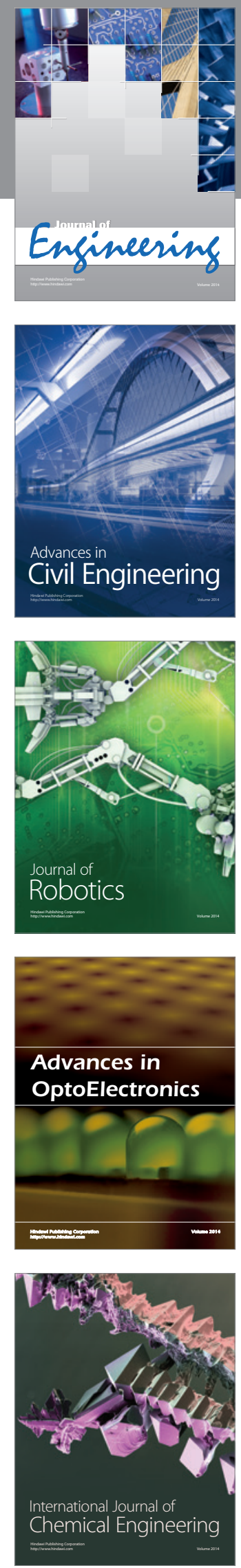

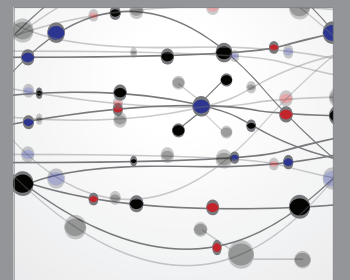

The Scientific World Journal
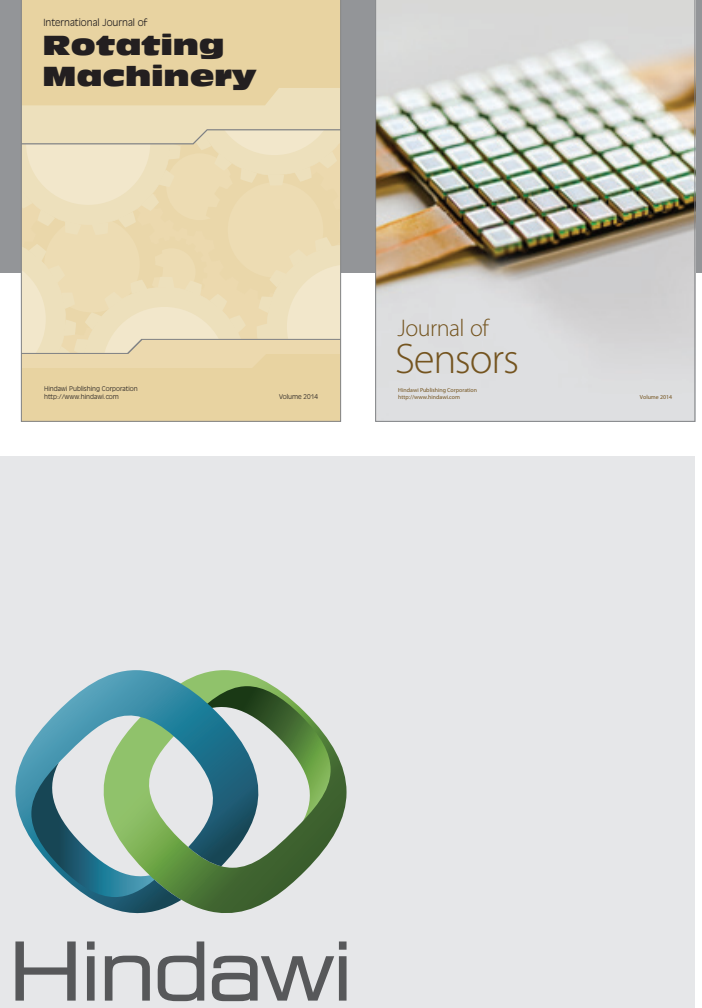

Submit your manuscripts at http://www.hindawi.com
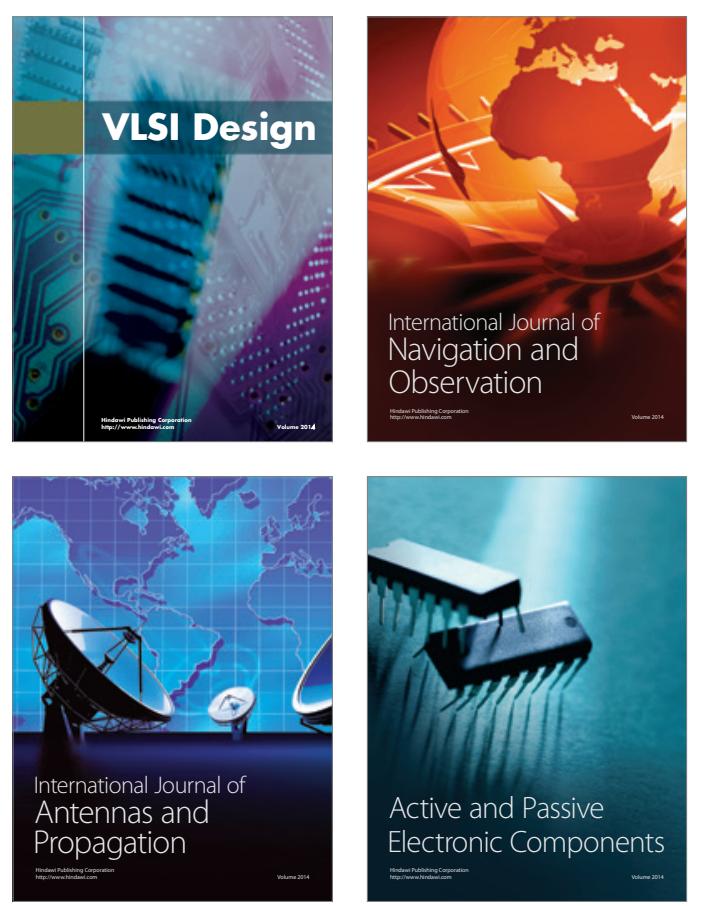
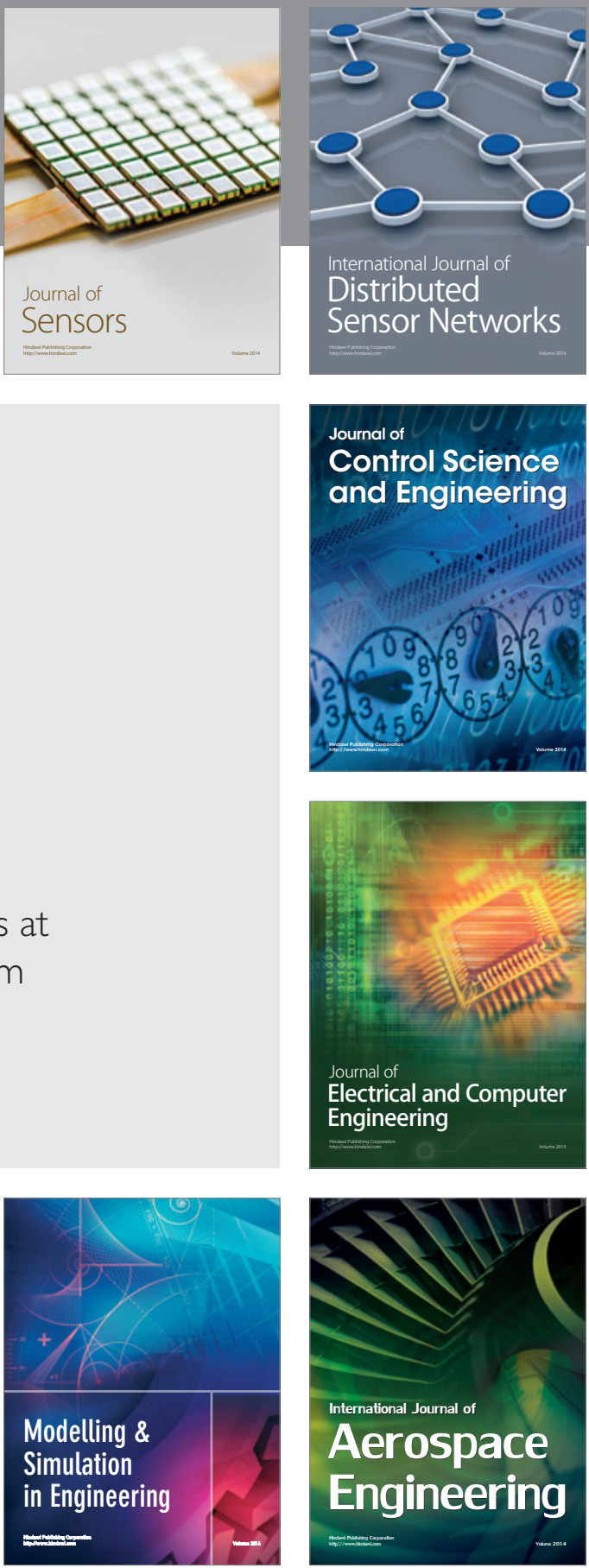

Journal of

Control Science

and Engineering
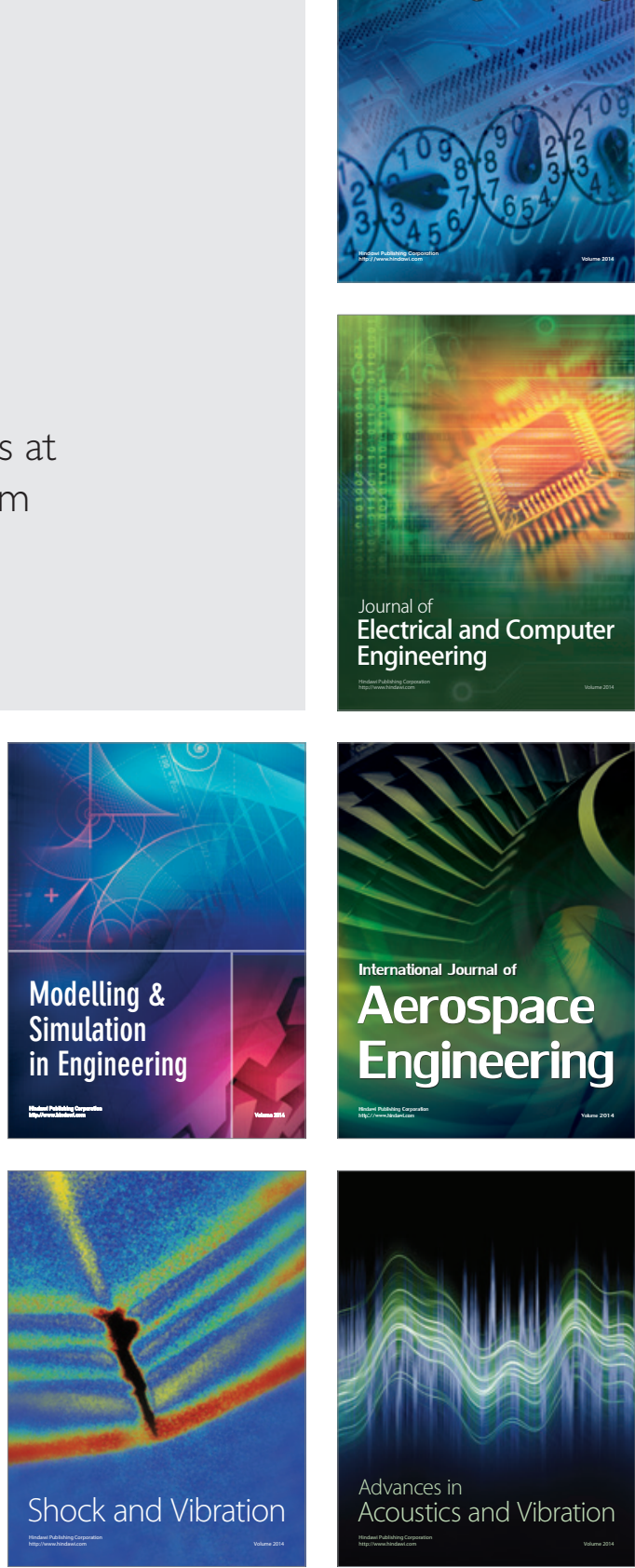